\title{
Biomass based porous carbon for supercapacitor by hydrothermal assisted activating method
}

\author{
Congcong Huang ${ }^{1, *}$, Yunhui Dong ${ }^{2}$ and Xingjun Dong ${ }^{3}$ \\ ${ }^{1}$ Department of Food Engineering, Shandong Business Institute, 264670 Yantai, P. R.China \\ ${ }^{2}$ School of Chemistry and Chemical Engineering, Shandong University of Technology, 255049 Zibo, P. R. China \\ ${ }^{3}$ Yantai Xinke Electronic Technology Co. LTD, 264000 Yantai, P. R.China
}

\begin{abstract}
A facile route has been employed to synthesize a series of high performance activated carbons as the electrode material for supercapacitors. The structure of the carbons are characterized by $\mathrm{N}_{2}$ adsorption/desorption and FTIR spectroscopy. The electrochemical performances of the carbons as an electrode material were evaluated by cyclic voltammetry test and galvanostatic charge/discharge measurements. As a biomass derived carbon, $\mathrm{KOH}-1$ exhibits high capacity, good rate capability and high energy density, indicating the promising application of hydrothermal combining with $\mathrm{KOH}$ activation method for biomass materials that used in supercapacitors.
\end{abstract}

\section{Introduction}

As the diminishing fossil fuels sources and emerging environmental issues, exploring alternative energy resources which are sustainable and renewable, like solar energy, wind power, tidal energy are urgently needed. Supercapacitors are of great interest due to the fact that they combine high power density with ultra-long cycle life and quick charge-discharge capability, properties that are currently lack in rechargeable batteries.

Undoubtedly, the successful commercial applications of supercapacitors are highly depended on suitable and superior electrode materials [1].Carbon materials are the most promising candidate owing to its high specific surface area, high conductivity and low cost. From the industry point of view, the large scale production of carbon materials in a sustainable way, renewable biomass based carbon is the primary feed stock.

Thus, recently a number of works have focused on using the by-products from food processing and agricultural industries as the precursors to prepare the carbon-based electrodes for supercapacitors. Various types of agricultural by-products like wheat straw [2], corncob [3], peanut shells [4] and rice husk [5] are in particularly interest because they are in the effort to reduce the production cost and preserve the environment.

Walnuts are the most productive nut in China with a yearly production of about 3.6 million tons [6]. Whereas the walnut shell are mainly used as fuels, which are the waste of resource. Consequently, there is a great need to convert walnut shells from waste to wealth. In this article, we used the walnut shell as the carbon precursor and a facile way was employed to develop a porous carbon with multiple organic functional groups which can be used in the supercapacitors.

\footnotetext{
* Corresponding author: huangcongcong@sdbi.edu.cn
}

\section{Experimental}

\subsection{Chemicals}

Walnut shell ( $<200 \mu \mathrm{m}), \mathrm{KOH}, \mathrm{ZnCl}_{2}$, distilled water.

\subsection{Preparation of carbon samples}

The walnut shell $(\mathrm{W})$ were sieved after mechanical crush to obtain particle sizes smaller than $200 \mu \mathrm{m}$. Then the hydrothermal method was used to pretreatment the samples, in a typical preparation, a specific amount of solid activating agent (A) was dissolved in distilled water followed by add into $2 \mathrm{~g}$ of walnut shell powder and then the mixture was transferred into a Teflon threaded stainless steel autoclave, followed by heating the autoclave to $150^{\circ} \mathrm{C}$ for $12 \mathrm{~h}$. Then the mixture was stirred at $100{ }^{\circ} \mathrm{C}$ to evaporate the water. When the mixture turned into muddy, transferred it into the tube furnace for activation at the temperature of $800{ }^{\circ} \mathrm{C}$ under $\mathrm{N}_{2}$. Obtained products were washed with excess amount of $6 \mathrm{M} \mathrm{HCl}$ under magnetic stirring and washed with distill water until the $\mathrm{pH}$ of the mixture was about 7.0 followed by filtration and drying. The prepared samples were denoted as $\mathrm{A}-\mathrm{X}$, where $\mathrm{X}$ represents the mass ratio of $\mathrm{A} / \mathrm{W}$. For example, $\mathrm{KOH}-3$ means the mass ratio of the $\mathrm{KOH}$ :walnut shell is $3: 1$.

\subsection{Physical characterization}

The porous structure of the carbon samples were examined by $\mathrm{N}_{2}$ sorption at $77 \mathrm{~K}$ using Micromeritics ASAP 2020 instrument. Specific surface areas were 
calculated by using the BET (Brunauer-Emmett-Teller) equation. The total pore volume $\left(\mathrm{V}_{\mathrm{T}}\right)$ was obtained at $\mathrm{P} / \mathrm{P}_{0}=0.995$, and the pore size distribution was calculated by the BJH (Barrett, Joyner, Halender) method using the desorption branch of the isotherm. Before the measurements, all samples were degassed at $350^{\circ} \mathrm{C}$ under vacuum for $4 \mathrm{~h}$. FTIR spectroscopy (Nexus912A, Thermo Nicolet, USA) was used to investigate the functional groups on the carbon surface.

\subsection{Electrode preparation and electrochemical evaluation}

Samples were grounded with $5 \mathrm{wt} \%$ of PTFE (polytetrafluoroethylene) binder and pressed between two pieces of nickel foam under $300 \mathrm{~kg} / \mathrm{cm}^{2}$. Two electrodes were divided by insulating fiber-cloth (with thickness of 300 micrometers) and tested by cyclic voltammetry and galvanostatic charge-discharge measurements in $\mathrm{CHI}$ 660C electrochemical testing system. Both electrodes containing comparable masses, ca. $20 \mathrm{mg}$ of carbon which were dried overnight at $100{ }^{\circ} \mathrm{C}$ under vacuum oven. The electrodes were impregnated with the electrolyte $(30 \mathrm{wt} \% \mathrm{KOH})$ under vacuum to guarantee the thorough wetting.

\section{Results and discussion}

\section{1 porous texture characterization}

The adsorption and desorption isotherms of the samples were shown in Figure 1, it can be clearly seen that all the isotherms except for $\mathrm{ZnCl}_{2}-3$ and $\mathrm{ZnCl}_{2}-4$ exhibit the typically I isotherms, which means they can get a large absorbent amount below the pressure of 0.1 and reach a steady platform without hysteresis loops. It can be concluded that the pore of the carbon samples were mainly consist of abundant micropore and a small amount of mesopore. As for the isotherms of $\mathrm{ZnCl}_{2}-3$ and $\mathrm{ZnCl}_{2}-4$, they show type-IV curves with hysteresis loops at relative pressure above 0.4 , which suggest the possession of abundant mesopore of the samples.

Pore-structure parameters of all studied carbons are listed in Table 1. It is found that the specific surface area reached a maximum value when the $\mathrm{A} / \mathrm{W}$ is 3 , further increasing the activation ratio, the $\mathrm{S}_{\mathrm{BET}}$ decreased which may be caused by the collapse of the adjacent micropore. $\mathrm{KOH}-3$ possesses the highest BET surface area, the micropore surface area and total pore volume, being $1862 \mathrm{~m}^{2} / \mathrm{g}, 1496 \mathrm{~m}^{2} / \mathrm{g}$ and $1.101 \mathrm{~cm}^{3} / \mathrm{g}$, respectively, which prove the effectiveness of $\mathrm{KOH}$ activation. We can assumed that the amount of mesopore and micropore can be controlled by adjusting the active agent ratio.

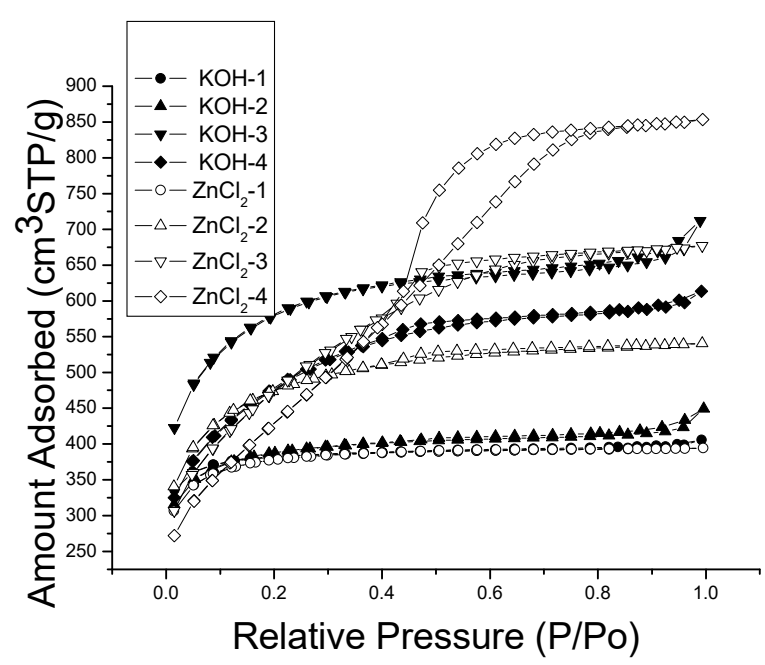

Figure 1. $\mathrm{N}_{2}$ sorption isotherms of carbon samples. Table 1.Surface area and pore-structure parameters of all samples.

\begin{tabular}{|c|c|c|c|c|c|}
\hline Sample & $\mathbf{A} /$ & $\begin{array}{c}\text { S } \text { BET } \\
\left(\mathbf{m}^{2} / \mathbf{g}\right)\end{array}$ & $\begin{array}{c}\mathbf{S}_{\text {meso }} \\
\left(\mathbf{m}^{\mathbf{2}} / \mathbf{g}\right)\end{array}$ & $\begin{array}{c}\mathbf{S}_{\text {micro }} \\
\left(\mathbf{m}^{\mathbf{2}} / \mathbf{g}\right)\end{array}$ & $\begin{array}{c}\mathbf{V}_{\mathbf{T}} \\
\left(\mathbf{c m}^{\mathbf{3}} \mathbf{g}\right)\end{array}$ \\
\hline $\mathrm{KOH}-1$ & $1: 1$ & 1165 & 59 & 1106 & 0.628 \\
\hline $\mathrm{KOH}-2$ & $2: 1$ & 1198 & 127 & 1071 & 0.695 \\
\hline $\mathrm{KOH}-3$ & $3: 1$ & 1862 & 366 & 1496 & 1.101 \\
\hline $\mathrm{KOH}-4$ & $4: 1$ & 1592 & 625 & 967 & 0.949 \\
\hline $\mathrm{ZnCl}_{2}-1$ & $1: 1$ & 1163 & 82 & 1081 & 0.61 \\
\hline $\mathrm{ZnCl}_{2}-2$ & $2: 1$ & 1498 & 332 & 1166 & 0.836 \\
\hline $\mathrm{ZnCl}_{2}-3$ & $3: 1$ & 1641 & 1038 & 603 & 1.048 \\
\hline $\mathrm{ZnCl}_{2}-4$ & $4: 1$ & 1549 & 1520 & 29 & 1.32 \\
\hline
\end{tabular}

\subsection{Electrochemical properties based on cyclic voltammetry measurements}

Figure 2 show the $\mathrm{CV}$ profiles of all samples at the scan rate of $20 \mathrm{mV} / \mathrm{s}$ and $100 \mathrm{mV} / \mathrm{s}$ in $30 \% \mathrm{KOH}$ electrolyte. At $20 \mathrm{mV} / \mathrm{s}$, all curves are standard rectangular shape which are typical for a stable double-layer capacitor within the voltage $0-0.9 \mathrm{~V}[1]$. At the scan rate of $100 \mathrm{mV} / \mathrm{s}$, the rectangular shape of all samples are maintained with only slight distortion, which shows the excellent capacitive behavior at high drain rate. The responded currents of the $\mathrm{KOH}$ activate carbons were larger than $\mathrm{ZnCl}_{2}$ activate carbons, suggesting that $\mathrm{KOH}$ is more effective as an activation agent. Nevertheless, the surface area and the percentage of mesopore is relatively low compare to $\mathrm{ZnCl}_{2}$ active samples, this phenomenon may be caused by the functional groups which generated by $\mathrm{KOH}$ activation. 

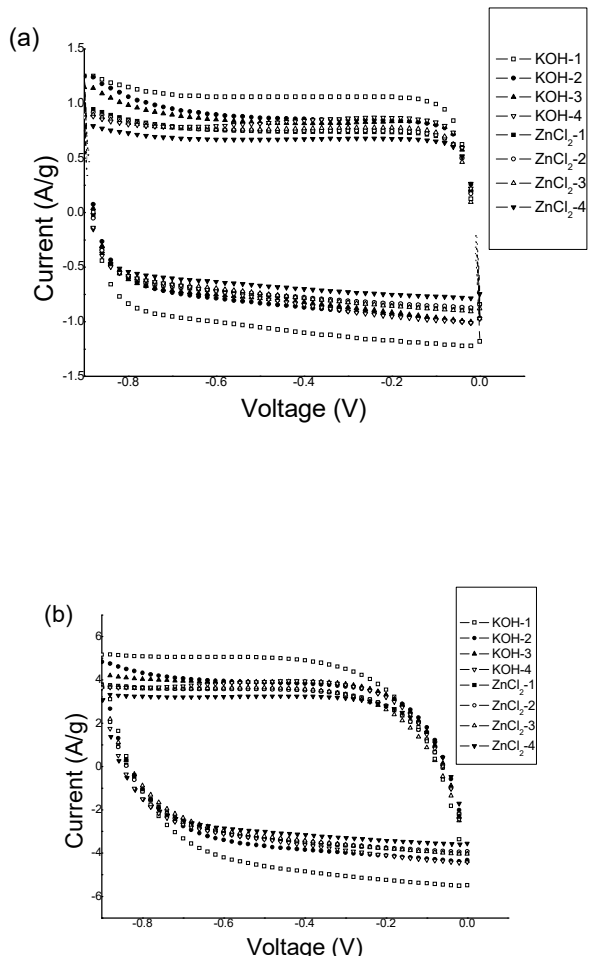

Figure 2. Cyclic voltammograms of all investigated samples recorded at the scan rates of $20 \mathrm{mV} / \mathrm{s}$ (a) and $100 \mathrm{mV} / \mathrm{s} \mathrm{(b)}$

\subsection{Galvanostatic charge/discharge tests Colour illustrations}

Galvanostatic charge-discharges with the current density of $20-200 \mathrm{~mA} / \mathrm{cm}^{2}$ were conducted to obtain the specific capacitances of carbon samples. The corresponding specific capacitances were calculated by the equation :

$$
C_{\text {sin } g l e}=\frac{I \times t}{\nabla V \times m}
$$

where $\mathrm{I}$ is the discharge current $(\mathrm{mA}), \mathrm{t}$ is the total discharge time (s), $\mathrm{m}$ is the total mass of active material in both electrodes $(\mathrm{g}), \mathrm{V}$ is the potential difference during the discharging (V) and Csingle is singleelectrode specific capacitance $(\mathrm{F} / \mathrm{g})$.

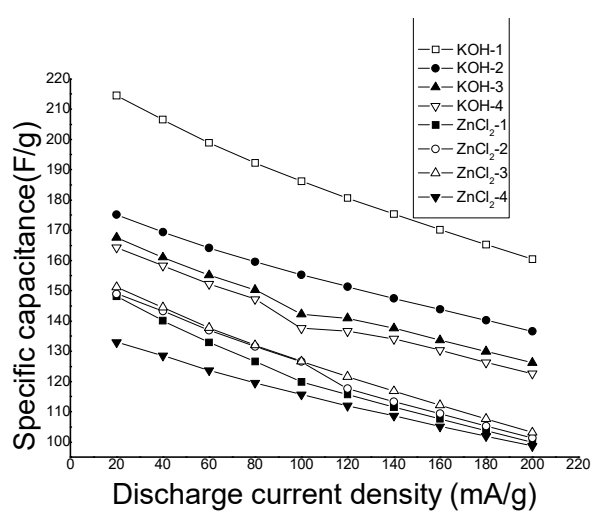

Figure 3. Comparison of capacitance decay with increasing discharge current density for all samples

As shown in Figure 3, KOH-1 shows the best performance and posses the largest specific capacitance of $215 \mathrm{~F} / \mathrm{g}$. At a low current density, the ions have enough time to diffuse into the micropore of the carbon, while at a higher current density the ions can only penetrate into part of micropores, which can explain why the capacitance of all the samples decrease as the current density increased gradually.

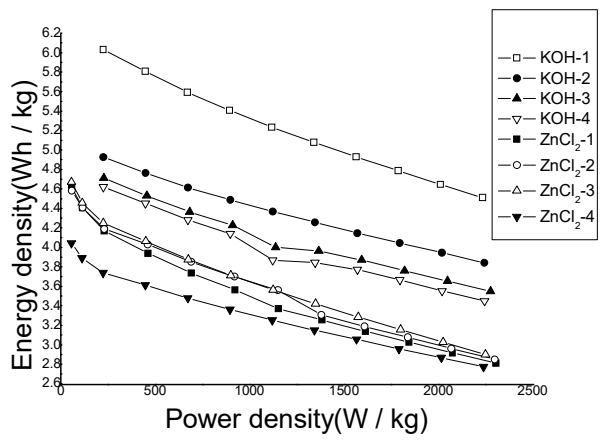

Figure 4. Ragone plots for all samples

The Ragone plots are frequently used in demonstration of power densities and energy densities of supercapacitors thus Figure 4 displays the plots for all investigated carbons. The energy density (in $\mathrm{Wh} / \mathrm{kg}$ ) is calculated as

$$
E=\frac{1}{2} C V^{2}
$$

where $\mathrm{C}$ is the capacitance of the two-electrode capacitor and $\mathrm{V}$ is the voltage decrease in discharge. The power (in $\mathrm{W} / \mathrm{kg}$ ) is calculated as

$$
P=\frac{2 E}{\Delta t_{d}}
$$

where $\mathrm{E}$ is the energy density and $\Delta \mathrm{td}$ is the time spent in discharge. 
In $30 \mathrm{wt} \% \mathrm{KOH}$ aqueous electrolyte, the energy density of $\mathrm{KOH}$ series carbons are higher than $\mathrm{ZnCl}_{2}$ series carbons at the same power density, which confirmed the effectiveness of $\mathrm{KOH}$ as an activation agent, However, at fast charge-discharge rates, only $\mathrm{KOH}-1$ exhibits stable performance which means $\mathrm{KOH}-$ 1 can release most of the energy under high discharge power conditions.

\subsection{Surface functional characterization}

It is obviously to see that $\mathrm{KOH}-1$ and $\mathrm{ZnCl}_{2}-1$ have the similar pore structure and pore size distribution according to Table 1, but the electric capacitive performance are quit different (as shown in the electrical measurement), this phenomena was speculate to be caused by the different surface chemistry or functional groups.

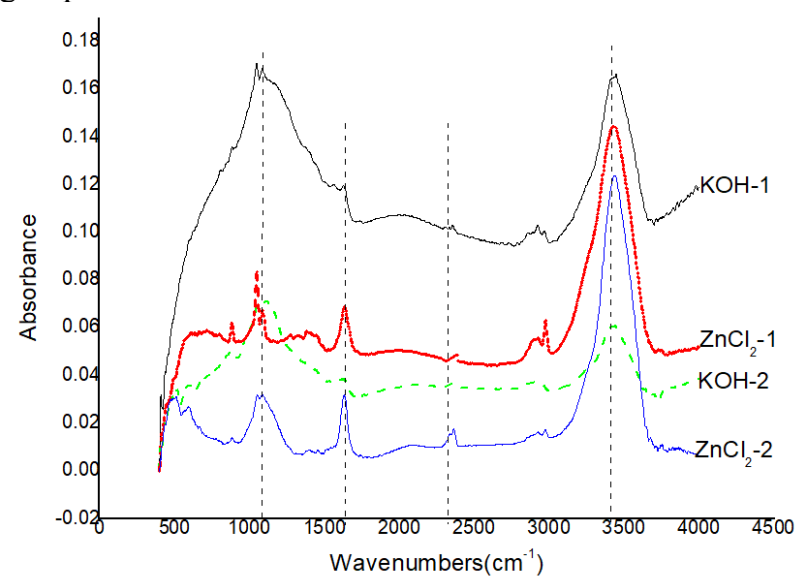

Figure5. FTIR spectra of $\mathrm{KOH}-1, \mathrm{KOH}-2, \mathrm{ZnCl}_{2}-1$ and $\mathrm{ZnCl}_{2}-$ 2

The FTIR spectra of $\mathrm{KOH}-1, \mathrm{KOH}-2, \mathrm{ZnCl}_{2}-1$ and $\mathrm{ZnCl}_{2}-2$ are shown in Figure 5. At the band of $1100 \mathrm{~cm}^{-1}$, samples $\mathrm{KOH}-1$ enhanced obviously compare to the other samples which was assigned to the $\mathrm{C}-\mathrm{O}$ stretching vibration. It was found that for the $\mathrm{ZnCl}_{2}-1$ and $\mathrm{ZnCl}_{2}-2$, new bands appeared at $1625 \mathrm{~cm}^{-1}$, assigned to bending vibration of $\mathrm{C}=\mathrm{O}$, the broad band at $3450 \mathrm{~cm}^{-1}$ assigned to N-H stretching vibration, which enhanced obviously compare to the $\mathrm{KOH}$ activated samples. At the band of $2360 \mathrm{~cm}^{-1}$, it was assigned to the $\mathrm{CO}_{2}$. The results suggested that the surface functional groups varied in different samples. The surface functional groups on the carbon can affect the wettability, polarity and stability of carbon materials. The increase of wettability will be beneficial to the affinity of carbon surface to electrolyte ions, which will increase the ability of carbon surface to adsorb electrolytes, thus improving the ability of carbon material surface to form electric double layer, and thus improving the specific capacitance of the supercapacitors.

\section{Conclusions}

In this work, a series of carbons were synthesized by using hydrothermal assisted chemical activation of the walnut shell, KOH-1 shows the best electrochemical behavior with a specific capacitance of $215 \mathrm{~F} / \mathrm{g}$ in 30 wt $\% \mathrm{KOH}$ electrolyte. It can be attributed to the enriched $\mathrm{C}-\mathrm{O}$ functional groups and large mesopore structure of this carbon. Similarly, this method may shed some light on tailoring more biomass carbon materials for low cost and high-performance supercapacitors.

\section{Acknowledgements}

We are grateful for the financial supports by A Project of Shandong Province Higher Educational Science and Technology Program (J18KB048), Campus integration development project of Yantai (2019XDRHXMPT32).

\section{References}

1. B.E.Conway, Electrochemical Supercapacitors ( Kluwer-Plenum, New York, 1999)

2. G. Gou , F. Huang, M. Jiang, et al.[J]. Renew. Energy, 149(2019)

3. Y. Shaoran, Z. Kaili, Nanomaterials, 8, 4(2018):181

4. N. Yadav, M. K. Singh, N. Yadav, et al, J. Power Sources, 402 (2018):133-146

5. Z. Chen, X. Wang, B.Xue, etal., Carbon, 161( 2020): 432-444

6. http://www.csnc.cn/hangyedongtai/20200806104056. html 